\title{
The effect of glutamine supplementation on hematopoietic stem cell transplant outcome in children: A case-control study
}

\begin{abstract}
Kuskonmaz B, Yalcin S, Kucukbayrak O, Cetin N, Cetin M, Tezcan I, Uckan D. The effect of glutamine supplementation on hematopoietic stem cell transplant outcome in children: A case-control study. Pediatr Transplantation 2008: 12: 47-51. ( 2008 Blackwell Munksgaard
\end{abstract}

Abstract: HSCT associated morbidity and mortality is usually attributed to high-dose chemotherapy/radiotherapy regimens used for conditioning. Glutamine (Gln), a conditionally essential amino acid during severe catabolic states, has been shown to have favorable effects in patients with malignancies and in those undergoing HSCT. However, controversy exists regarding its routine use. Studies in children investigating gln supplementation are very limited. In the present study, including 21 gln-supplemented and 20 control pediatric patients, gln supplementation was shown to reduce the duration of fever and decrease the incidence of SOS during the HSCT course. In addition, a decrease in drug-related toxicity and a trend toward reduced incidence of severe mucositis were observed.

\author{
Baris Kuskonmaz ${ }^{1}$, Songul Yalcin ${ }^{2}$ \\ Ozlem Kucukbayrak ${ }^{1}$, Nevin Cetin $^{3}$, \\ Mulla Cetin ${ }^{1}$, Ilhan Tezcan ${ }^{4}$ and Duygu \\ Uckan $^{3}$ \\ ${ }^{1}$ Department of Pediatric Hematology, ${ }^{2}$ Department \\ of Social Pediatrics, ${ }^{3}$ Pediatric Bone Marrow, \\ Transplantation Unit, ${ }^{4}$ Department of Pediatric \\ Immunology, Hacettepe University Faculty of \\ Medicine Ihsan, Dogramaci Childrens Hospital, \\ Ankara, Turkey \\ Key words: hematopoietic stem cell transplantion - \\ children - glutamine \\ Duygu Uckan, Pediatric Bone Marrow Transplantation \\ Unit, Hacettepe University Faculty of Medicine Ihsan, \\ Dogramaci Childrens Hospital, Ankara, Turkey \\ Tel.: +90 3123051979 \\ Fax: +903123243228 \\ E-mail: duckan@hacettepe.edu.tr \\ Accepted for publication 14 June 2007
}

Although HSCT is curative in many diseases, toxicities of organ systems, mainly gastrointestinal toxicity manifesting with mucositis and diarrhea requiring TPN, may necessitate additional supportive care strategies (1). Glutamine $(\mathrm{Gln})$ is a critical substrate in many key metabolic processes, including inter-organ nitrogen transfer, protein and nucleic acid synthesis, glyconeogenesis, and acid-base homeostasis. Gln is also used as a major fuel and/or substrate by intestinal mucosal cells, lymphocytes, and other immune cells $(2,3)$. During catabolic states, gln concentrations in intracellular pools (primarily skeletal muscle) fall rapidly because gln is used for renal ammoniogenesis and serves as an oxidizable fuel for stimulated lymphocytes, macrophages and

Abbreviations: aGVHD, acute GVHD; BMT, bone marrow transplantation; CMV, cytomegalovirus; Gln, glutamine; GVHD, graft vs. host disease; HLA, human leukocyte antigen; HSCT, hematopoietic stem cell transplantation; OR, odds ratio; SOS, sinusoidal obstruction syndrome; TBI, total body irradiation; TPN, total parenteral nutrition. intestinal mucosal cells (4-7). Although gln is not strictly an essential amino acid, it is considered to be conditionally essential during severe catabolic illness (8). It has been shown that the hypercatabolic state following chemotherapy is associated with low gln state (9). The use of gln-supplemented nutrition has received increasing attention in basic, translational, and clinical research in HSCT patients (10).

High-dose chemotherapy/radiotherapy is commonly used in the HSCT setting (10). Gln has been shown to prevent chemotherapy/radiotherapy-induced toxicity in patients with malignancies in experimental and clinical studies. The favorable effect has been shown mainly in the gastrointestinal system. In addition, chemotherapy-induced neuropathy, cardiotoxicity, myalgias, and arthralgias have been reduced with gln supplementation (11-13). In an experimental study in rodents, gln supplementation has been shown to enhance the selectivity of anti-tumor drugs by protecting normal tissues from and possibly sensitizing tumor cells to chemotherapy 
treatment-related injury (14). The initial clinical safety and efficacy of gln administration in adult HSCT patients was reported in 1990 and 1992 $(15,16)$.

However, the role of gln in parenteral nutrition remains a controversial issue, a decade since the introduction of gln-enriched TPN (17). Also, studies about gln supplementation in children alone are limited. To the best of our knowledge, there is only one randomized study in the literature consisting of pediatric patients alone in which a favorable effect of oral gln on mucositis was demonstrated (18). Considering the difficulties in intake of oral medications in children, particularly those undergoing transplantation, we used parenteral gln to determine the effect of gln supplementation not only on mucositis but other HSCT complications as well.

\section{Patients and materials}

The study compared the results of the two different treatment regimens at Hacettepe University Ihsan Dogramaci Children's Hospital, Bone Marrow Transplantation Unit. We started gln supplementation as Dipeptiven (Fresenius Kabi, Graz, Australia) at a dose of $0.4 \mathrm{~g} / \mathrm{kg} /$ day in January 2004 from day -9 till day 21 through central venous catheter with or without TPN solution. After this period, 21 cases who underwent allogeneic HSCT and had cytotoxic-conditioning regimen received gln supplementation (supplemented group) until the study point in January 2005. For each supplemented case, a control-case comparable with respect to donor type, diagnosis and age distribution was selected from the presupplemented period (control group). For one supplemented case, no suitable control-case was found.

Busulfan-based conditioning regimens were used in most patients; TBI was used in five patients. Infection prophylaxis included fluconazole for antifungal prophylaxis and acyclovir for antiviral prophylaxis, trimethoprim-sulfamethoxazole, and ciprofloxacin for bacterial prophylaxis. Weekly intravenous immunoglobulin was administered in all patients. Cyclosporin $\mathrm{A} \pm$ short-term methotrexate was used as GVHD prophylaxis. TPN was started following a five-day period of decreased oral intake $(<50 \%$ percent of recommendended calories) or when weight loss exceeded $10 \%$ during treatment.

Myeloid engraftment was defined as the first of three consecutive days when the neutrophil count was higher than $0.5 \times 10^{9} / \mathrm{L}$. Patients who did not engraft, as well as those with transient engraftment of donor cells, were considered to have graft failure. Drug-related adverse effects were evaluated in both groups, and included clinical descriptive features attributed to drug use such as parotitis, toxic hepatitis, cardiotoxicity, neurotoxicity, hemorrhagic cystitis, drug eruption, urticarial rash, serum sickness, and anaphylaxis. aGVHD and veno-occlusive disease/SOS were diagnosed and graded according to conventional criteria $(19,20)$.

Documented infection was defined as microbiologically confirmed systemic, disseminated, invasive, rapidly progressive infection, or catheter-related infection, or pneumonia diagnosed by clinical and radiological findings, or CMV disease (CMV infection with CMV organ involvement). The following incidents were excluded: commonly encountered skin contamination bacteria (e.g., coagulase-negative staphylococci) detected in a single blood culture, catheter site local infection except tunnel infection. Statistical analysis was performed by SPSS (SPSS Inc., Chicago, IL, USA). Chi-square (or Fisher's exact test) and Student's $t$-test (or Mann-Whitney $U$ test) were used for statistical comparisons where appropriate.

\section{Results}

The groups were comparable with respect to donor type, age distribution, gender, HLA match, diagnosis (malignant and non-malignant; Table 1). In addition, the groups had taken similar conditioning regimens and GVHD prophylaxis. The number of patients who received fresh frozen plasma, albumin infusions, and TPN was comparable in the supplemented and control groups. The number of patients with neutrophil engraftment was similar in the supplemented and control groups [19/21 (90\%) and 18/20 (90\%), respectively, Table 2]. The date of neutrophil engraftment was also comparable in the two groups $(15.0 \pm 3.4$ and $14.6 \pm 3.3$ days, respectively).

Although the ratio of patients who developed fever was similar in both groups, the duration of fever was significantly decreased in the supplemented group when compared with the control

Table 1. Baseline characteristics of patients*

\begin{tabular}{|c|c|c|}
\hline & $\begin{array}{l}\text { Supplemented } \\
\text { group, } \mathrm{n}(\%)\end{array}$ & $\begin{array}{l}\text { Control } \\
\text { group, n (\%) }\end{array}$ \\
\hline $\mathrm{n}$ & 21 & 20 \\
\hline Age, yr (mean \pm s.d.) & $8.30 \pm 5.20$ & $6.90 \pm 4.30$ \\
\hline $\operatorname{Sex}(M / F)$ & $16 / 5$ & $15 / 5$ \\
\hline Malignant diseases & $10(48)$ & $11(55)$ \\
\hline $\mathrm{CML}$ & $4(19)$ & $4(20)$ \\
\hline AML & $4(19)$ & $2(10)$ \\
\hline ALL & $2(10)$ & $1(5)$ \\
\hline Myelodysplastic syndrome & - & 3 \\
\hline SCID + AML & - & $1(5)$ \\
\hline Non-malignant diseases & $11(52)$ & $9(45)$ \\
\hline Wiskott-Aldrich syndrome & $1(5)$ & \\
\hline Leukocyte adhesion deficiency & $1(5)$ & \\
\hline Griscelli syndrome & $2(10)$ & \\
\hline Adrenoleukodystrophy & $1(5)$ & $2(10)$ \\
\hline Mannosidosis & & $1(5)$ \\
\hline Osteopetrosis & & $1(5)$ \\
\hline Fanconi aplastic anemia & $2(10)$ & $1(5)$ \\
\hline Acquired aplastic anemia & $1(5)$ & $1(5)$ \\
\hline Thalassemia major & $3(14)$ & $3(15)$ \\
\hline HLA $6 / 6$ matched donor & $19(91)$ & $17(85)$ \\
\hline ABO major incompatibility & $2(10)$ & $2(10)$ \\
\hline
\end{tabular}

s.d., standard deviation; $\mathrm{M}$, male; F, female; CML, chronic myeloid leukemia; AML, acute myeloid leukemia; ALL, acute lymphoblastic leukemia; SCID, severe immune deficiency; HLA, human leukocyte antigen. ${ }^{*} p>0.05$. 


\begin{tabular}{lccc}
\hline & $\begin{array}{c}\text { Supplemented } \\
\text { group }(\mathrm{n}=21)\end{array}$ & \multicolumn{1}{c}{$\begin{array}{c}\text { Control group } \\
(\mathrm{n}=20)\end{array}$} \\
\hline Patients who received TPN & $14(67)$ & $12(60)$ & $18(90)$ \\
Neutrophil engraftment & $19(90)$ & $14.6 \pm 3.3$ & $17(85)$ \\
Neutrophil engraftment date after BMT (days) & $15.0 \pm 3.4$ & $12.9 \pm 11.2$ & 0.920 \\
Incidence of fever & $19(91)$ & $11 / 20(55.0)$ & 0.727 \\
Duration of fever (days) & $5.74 \pm 4.5$ & $6(30)$ & 0.021 \\
Documented infection & $8(38.1)$ & $3(15)$ & 0.278 \\
aGVHD & $5(24)$ & $7(35)$ & 0.734 \\
aGVHD grade 3-4 & $1(5)$ & $4(20)$ & 0.343 \\
SOS & $2(10)$ & $8(55)$ & 0.067 \\
SOS moderate-severe & $2(10)$ & $6(40)$ & 0.410 \\
Mucositis, grade 3-4 & $6(29)$ & $5(25)$ & 0.118 \\
Drug-related toxicity & $3(14)$ & $8(40)$ & 0.085 \\
Discharge $<$ day 37 & $12(57)$ & 0.300 \\
Mortality before discharge & $2(10)$ & 0.238 \\
Overall mortality & $6(29)$ & 0.440 \\
\hline
\end{tabular}

Values are expressed as $\mathrm{n}(\%)$ or mean \pm s.d.

TPN, total parenteral nutrition; BMT, bone marrow transplantation; aGVHD, acute graft vs. host disease; SOS, sinusoidal obstruction syndrome.

group $[5.74 \pm 4.45$ and $12.94 \pm 11.17$, respectively, $\mathrm{p}=0.021]$. In cases with fever, total febrile days over seven were seen less frequently in the supplemented group $(3 / 19,15.8 \%)$ than in the control group $(9 / 17,52.9 \%$; OR: $0.167,95 \%$ CI: $0.035-0.792, p=0.018)$. Documented infection was present in eight of 21 patients $(38.1 \%)$ in the supplemented group vs. $11 / 20(55.0 \%)$ in the control group. There was no statistically significant difference in the rate of aGVHD and grade 3-4 aGVHD in the supplemented and control groups (Table 2).

Patients receiving gln supplementation had lower incidence of SOS ( $\mathrm{p}=0.067)$. The SOS incidence was two of 21 patients $(10 \%)$ in the supplemented and seven of 20 patients $(35 \%)$ in the control groups. There was a decreased risk of SOS associated with use of gln (OR: $0.195,95 \%$ CI: 0.035-1.095). Although the incidence of grade 3-4 mucositis was lower in the supplemented patients, the difference was not statistically significant (Table $2, \mathrm{p}=0.118$ ).

Patients in the supplemented group had a lower drug-related toxicity rate than patients in the control group $[3 / 21(14 \%) ; 8 / 20 \quad(40 \%)$, respectively; $\mathrm{p}=0.085]$. OR for drug-related toxicity was $0.25(95 \% \mathrm{CI}: 0.055-1.137)$. The median duration of hospitalization was similar in both groups. Twelve of 21 patients were discharged before 37 days (median day of discharge) following HSCT in the supplemented group whereas six of 20 were discharged before that day in the control group $(\mathrm{p}>0.05)$. Although the mortality before discharge and overall mortality rates after HSCT were lower (10\% and $29 \%$, respectively) in the supplemented group when compared with the control group (25\% and $40 \%$, respectively), the difference was not statistically significant. No adverse effect related to gln administration was noted.

\section{Discussion}

In the present study, the duration of fever was found to be significantly decreased in the glnsupplemented pediatric HSCT patients when compared with those not supplemented. However, there was no statistically difference in the incidence of documented infection between two groups. Ziegler et al. have reported beneficial effect of gln supplementation on the incidence of infection and fever in patients undergoing BMT. Schloreb and Amare's study does not suggest a favorable effect of gln on the incidence of fever or clinical infection and colonization $(16,21)$. The favorable effects of gln on immune functions, on the repair of mucosal barrier defenses (i.e., the gastrointestinal tract), or on the maintenance of tissue antioxidant stores have all been suggested as contributory factors involved in the decreased incidence of infection or fever in gln-supplemented patients (16).

Glu supplementation has been suggested to play a favorable role in hepatic protection from SOS both as a protective and possibly a therapeutic agent (22). The present study has shown that the ratio of patients who developed SOS was borderline significantly lower in the gln group than in the control group (OR: 0.195, $\mathrm{p}=0.067)$. The maintenance of hepatic GSH concentration has been suggested to protect the hepatocytes from the oxidant stress of high-dose 
conditioning regimens (22). Several reports suggest beneficial effects of gln supplementation in transplant patients in protection from $\operatorname{SOS}(8,9$, 23).

Gln administration has been shown to decrease the duration and severity of mucositis in pediatric and adult patients receiving doxorubicin chemotherapy for solid cancers in a doubleblind, crossover study. Potential benefits of oral gln have been suggested in BMT-associated mucositis as well (24). We found that, although not statistically significant, the ratio of patients who had grade 3-4 mucositis was lower in the supplemented group than in the control group. Data indicate that gln administration can decrease gastrointestinal toxicity to chemotherapy in cancer patients (24). However, controversial results exist in the literature. Four randomized controlled trials and an additional controlled trial have reported that i.v. and/or oral L-gln did not influence incidence or severity of mucositis after BMT (1, 16, 21, 25, 26).

Previous studies have suggested gln use to prevent chemotherapy/radiotherapy-induced toxicities in patients with malignancies. In the present study, drug-related toxicity was decreased (OR: 0.25) in transplanted children, and this is in agreement with previous studies $(27,28)$.

The clinical trials reporting the effects of gln on mortality in BMT patients with malignancies have shown controversial results $(16,21,29)$. In the present study, the overall survival rate was higher in the supplemented group than in the control group; however, it remained statistically insignificant perhaps because of the small numbers of patients. In our study, hospital stay above 37 days was also reduced in the gln-supplemented patients. Studies have shown that the cost of hospital care and length of stay were significantly decreased with gln supplementation, primarily because of decreased room and board costs $(16,21,30)$.

A positive effect of gln on recovery from neutropenia has been suggested (17) by increasing its availability for bone marrow precursor cells (31); however, clinical data have not supported this hypothesis (32), as shown in our study.

In conclusion, gln supplementation has been found to be associated with decreased duration of fever and decreased incidence of SOS, both of which may play a role towards improved survival rate. Additional studies with large numbers of cases are needed to determine the effect of gln on other transplant-related events.

\section{References}

1. Coghlin Dickson TM, Wong RM, Offrin RS, et al. Effect of oral glutamine supplementation during bone marrow transplantation. J Parenter Enteral Nutr 2000: 24: 61-66.

2. SAUBA WW. Intestinal glutamine metabolism and nutrition. J Nutr Biochem 1993: 4: 2-9.

3. Ziegler TR, Puckett AB, Griffiths DP, Galloway JR. Interactions between nutrients and growth factors in cellular growth and tissue repair. In: Ziegler TR, Pierce GF, Herdon DN, eds. Growth Factors and Wound Healing: Basic Science and Potential Clinical Applications. New York: Springer-Verlag, 1997: pp. 104-150.

4. Wilmore DW, Black PR, Muhlbacher F. Injured man: Trauma and sepsis. In: WinTERs RW, ed. Nutritional Support of the Seriously Ill Patient. New York: Academic Press, 1983: pp. 33-52.

5. Welbourne TC. Interorgan glutamine flow in metabolic acidosis. Am J Physiol 1987: 253: 1069-1076.

6. Windmueller HG. Glutamine utilization by the small intestine. Mol Biol 1982: 53: 201-238.

7. Newsholme EA, Newsholme P, Curi R, Challoner E, Ardawi MS. A role for muscle in the immune system and its importance in surgery, trauma, sepsis and burns. Nutrition 1988: 4: 261-268.

8. Brown SA, Goringe A, Fegan C, et al. Parenteral glutamine protects hepatic function during bone marrow transplantation. Bone Marrow Transplant 1998: 22: 281-284.

9. Goringe AP, Brown S, O'Callaghan U, et al. Glutamine and vitamin $\mathrm{E}$ in the treatment of hepatic veno-occlusive disease following high-dose chemotherapy. Bone Marrow Transplant 1998: 21: 829-832.

10. ZIEGLER TR. Glutamine supplementation in cancer patients receiving bone marrow transplantation and high dose chemotherapy. J Nutr 2001: 131(Suppl.): 2578-2584.

11. Sikov W, Akerley W, Strenger R, Cummings F. Weekly high-dose paclitaxel demonstrates significant activity in advanced breast cancer (abstract). Proc Am Soc Clin Oncol 1998: 17: 112a.

12. CaO Y, Kennedy R, Klimberg VS. Glutamine protects against doxorubicin-induced cardiotoxicity. J Surg Res 1999: 85: 178182.

13. Savarese D, Boucher J, Corey B. Glutamine treatment of paclitaxel-induced myalgia and arthralgias. J Clin Oncol 1998: 16: 3918-3919.

14. Rouse K, Nwokedi E, Woodliff Je, Epstein J, Klimberg VS. Glutamine enhances selectivity of chemotherapy through changes in glutathione metabolism. Ann Surg 1995: 221: 420426.

15. Ziegler TR, Benfell K, Smith RJ, et al. Safety and metabolic effects of L-glutamine administration in humans. J Parenter Enteral Nutr 1990: 14(Suppl.): 137-146.

16. Ziegler TR, Young LS, Benfell K, et al. Clinical and metabolic efficacy of glutamine-supplemented parenteral nutrition after bone marrow transplantation. A randomized, doubleblind, controlled study. Ann Intern Med 1992: 116: 821-828.

17. Scheid C, Hermann K, Kremer G, et al. Randomized, double-blind, controlled study of glycyl-glutamine-dipeptide in the parenteral nutrition of patients with acute leukemia undergoing intensive chemotherapy. Nutrition 2004: 20: 249-254.

18. Aquino VM, Harvey AR, Garvin JH, et al. A double-blind randomized placebo-controlled study of oral glutamine in the prevention of mucositis in children undergoing hematopoietic stem cell transplantation: A pediatric blood and marrow transplant consortium study. Bone Marrow Transplant 2005: 36: 611-616. 
19. Przepiorka D, Weisdorf D, Martin P, et al. 1994 consensus conference on acute GVHD grading. Bone Marrow Transplant 1995: 15: 825-828.

20. Mcdonald GB, Hinds MS, Fisher LD, et al. Veno-occlusive disease of the liver and multiorgan failure after bone marrow transplantation: A cohort study of 355 patients. Ann Intern Med 1993: 118: 255-267.

21. Schloerb PR, Amare M. Total parenteral nutrition with glutamine in bone marrow transplantation and other clinical applications (a randomized, double-blind study). J Parenter Enteral Nutr 1993: 17: 407-413.

22. Muscaritoli M, Grieco G, Capria S, iori AP, Rossi FANELLI F. Nutritional and metabolic support in patients undergoing bone marrow transplantation. Am J Clin Nutr 2002: 75: 183-190.

23. Nattakom TV, Charlton A, Wilmore DW. Use of vitamin E and glutamine in the successful treatment of severe venoocclusive disease following bone marrow transplantation. Nutr Clin Pract 1995: 10: 16-18.

24. Ziegler TR. Glutamine supplementation in bone marrow transplantation. Br J Nutr 2002: 87(Suppl. 1): 9-15.

25. Schloerb PR, Skikne BS. Oral and parenteral glutamine in bone marrow transplantation: A randomized, double-blind study. J Parenter Enteral Nutr 1999: 23: 117-122.
26. Jebb SA, Marcus R, Elia M. A pilot study of oral glutamine supplementation in patients receiving bone marrow transplants. Clin Nutr 1995: 14: 162-165.

27. Savarese DM, Savy G, Vahdat L, Wischmeyer PE, Corey B. Prevention of chemotherapy and radiation toxicity with glutamine. Cancer Treat Rev 2003: 29: 501-513.

28. Rubio IT, Cao Y, Hutchins LF, Westbrook KC, Klimberg VS. Effect of glutamine on methotrexate efficacy and toxicity. Ann Surg 1998: 227: 772-777 (Discussion 778-780).

29. Anderson PM, Ramsay NK, Shu XO, et al. Effect of low-dose oral glutamine on painful stomatitis during bone marrow transplantation. Bone Marrow Transplant 1998: 22: 339-344.

30. Macburney M, Young LS, Ziegler TR, Wilmore DW. A cost-evaluation of glutamine-supplemented parenteral nutrition in adult bone marrow transplant patients. J Am Diet Assoc 1994: 94: 1263-1266.

31. Coutinho L, Gilleece M, de Wynter E, Will A, Testa N. Clonal and long-term cultures using human bone marrow. In: Testa N, Molineux G, eds. Heamopoiesis: A Practical Approach. Oxford: Oxford University Press, 1993: pp. 75.

32. Ziegler tr, Bye RL, Persinger RL, Young LS, Antin JH, WILMORE DW. Effects of glutamine supplementation on circulating lymphocytes after bone marrow transplantation: A pilot study. Am J Med Sci 1998: 315: 4-10. 